\title{
Hemodynamic and pathophysiological characteristics of intradialytic blood pressure elevation in patients with end-stage renal disease
}

\author{
Kazuki Oosugi ${ }^{1}$, Naoki Fujimoto ${ }^{2}$, Kaoru Dohi ${ }^{1}$, Hirofumi Machida ${ }^{3}$, Katsuya Onishi ${ }^{1}$, Misao Takeuchi ${ }^{3}$, \\ Shinsuke Nomura ${ }^{1}$, Hideyuki Takeuchi ${ }^{3}$, Tsutomu Nobori ${ }^{2}$ and Masaaki Ito ${ }^{1}$
}

An increase in systolic blood pressure (SBP) after hemodialysis (intradialytic-HTN) is associated with adverse outcomes in patients on regular hemodialysis. However, the hemodynamic and Doppler echocardiographic characteristics of intradialytic-HTN and its impact on clinical outcomes are unclear. A retrospective analysis of 84 patients (45 men, $70 \pm 9$ years) stratified into three groups on the basis of SBP response from pre- to post-hemodialysis: $G_{H T N}$ (intradialytic-HTN, SBP increase $\geqslant 10 \mathrm{~mm} \mathrm{Hg}$ ), $G_{D R O P}<15 \mathrm{~mm} \mathrm{Hg}$ (SBP drop $<15 \mathrm{~mm} \mathrm{Hg}$ ), and $\mathrm{G}_{\mathrm{DROP}} \geqslant 15 \mathrm{~mm} \mathrm{Hg}$ (SBP drop $\geqslant 15 \mathrm{~mm} \mathrm{Hg}$ ). Hemodynamic and echocardiographic assessments were performed pre- and post-hemodialysis, and patients were followed for $41 \pm 17$ months. $G_{H T N}$ had higher blood glucose and lower baseline SBP, serum potassium and total cholesterol. Cardiothoracic ratio was smaller, and peak early diastolic mitral annular velocity $\left(E^{\prime}\right)$ was lower in $G_{H T N}$. During hemodialysis, SBP and diastolic blood pressure increased only in $G_{H T N}$. After hemodialysis, left ventricular (LV) filling pressure (E/E' ratio) decreased only in $G_{D R O P} \geqslant 15 \mathrm{~mm} \mathrm{Hg}$, resulting in a higher $E^{\prime} E^{\prime}$ ratio in $G_{H T N}$ than $G_{D R O P} \geqslant 15 \mathrm{~mm} \mathrm{Hg}$. Multivariate logistic regression analysis revealed a positive correlation between blood glucose and intradialytic-HTN, whereas cardiothoracic ratio, pre-hemodialysis SBP and the change in $\mathrm{E} / \mathrm{E}^{\prime}$ ratio with hemodialysis were negatively related to intradialytic-HTN. During follow-up, $G_{H T N}$ had more cardiovascular deaths than $G_{D R O P} \geqslant 15 \mathrm{~mm} \mathrm{Hg}(P=0.03)$. Multivariate Cox regression analysis showed that lower serum potassium and previous coronary artery disease, but not intradialytic-HTN, were associated with cardiovascular deaths. A higher LV afterload and elevated filling pressures after hemodialysis, indicative of increased cardiovascular stiffening and impaired diastolic filling, may contribute in part to an increased cardiovascular burden in patients with intradialytic-HTN. Hypertension Research (2014) 37, 158-165; doi:10.1038/hr.2013.123; published online 19 September 2013

Keywords: diastolic function; hemodialysis; intradialytic hypertension

\section{INTRODUCTION}

Hypertension promotes cardiovascular stiffening, ${ }^{1,2}$ which impairs left ventricular (LV) diastolic function, ${ }^{3}$ and contributes to an overall increased risk for cardiovascular comorbidities such as heart failure, myocardial infarction and stroke. ${ }^{4}$ Hypertension is an extremely common $(\sim 90 \%)$ syndrome in patients with end-stage renal disease on hemodialysis, ${ }^{5}$ and contributes to a higher risk of cardiovascular morbidity and mortality in these patients. ${ }^{6,7}$

During hemodialysis, excess fluid is removed by ultrafiltration, usually resulting in a reduction in blood pressure (BP). However, in some patients systolic BP (SBP) actually increases pre- to post-dialysis (intradialytic-HTN) despite a reduction in LV preload and body mass by fluid removal. ${ }^{8,9}$ Although it has been reported that intradialyticHTN is associated with an increased risk of mortality, ${ }^{10-12}$ the pathophysiological mechanisms contributing to intradialytic-HTN are complex and not well understood. Cardiovascular stiffening increases arterial BP, elevates LV filling pressure and afterload, and impairs LV diastolic function. Therefore, it is possible that profound cardiovascular stiffening and impaired LV diastolic function may contribute in part to an increased cardiovascular burden in patients with intradialytic-HTN. However, to date, no studies have evaluated hemodynamics and LV diastolic function in dialysis patients with and without intradialytic-HTN to support this contention. Such information is valuable as it may enable a better understanding of pathophysiological hallmarks of intradialytic-HTN and allow for the design of more optimal therapeutic options to forestall cardiovascular risk in these patients.

Therefore, the objectives of this study were twofold. First, we aimed to assess hemodynamics and LV diastolic function before and after hemodialysis, to elucidate the pathophysiological characteristics of intradialytic-HTN. Second, we aimed to determine whether intradialytic-HTN is associated with increased cardiovascular and

${ }^{1}$ Department of Cardiology and Nephrology, Mie University Graduate School of Medicine, Tsu, Japan; ${ }^{2}$ Department of Molecular and Laboratory Medicine, Mie University Graduate School of Medicine, Tsu, Japan and ${ }^{3}$ Department of Internal Medicine, Syojunkai Takeuchi Hospital, Tsu, Japan

Correspondence: Dr N Fujimoto, Department of Molecular and Laboratory Medicine, Mie University Graduate School of Medicine, 2-174 Edobashi, Tsu 514-8507, Japan. E-mail: naokifujimo@clin.medic.mie-u.ac.jp

Received 21 June 2013; revised 30 July 2013; accepted 2 August 2013; published online 19 September 2013 
noncardiovascular mortality in patients on regular hemodialysis. We hypothesized that advanced cardiovascular stiffening and impaired LV diastolic function contribute to the pathophysiology of intradialyticHTN

\section{METHODS}

\section{Patient population}

Eighty-four patients (45 men, age range 21-77 years) with end-stage renal disease, who had undergone regular hemodialysis in Mie University Hospital and Takeuchi Hospital, were enrolled in the present study between July and August 2008. Patients were excluded if they had atrial flutter/fibrillation, recent myocardial infarction, prior coronary artery bypass graft, LV ejection fraction $<50 \%$ by two-dimensional echocardiography, severe valvular heart disease, signs of infection, malignancy or previous habitual exercise defined as $\geqslant 30 \mathrm{~min}$, three times per week. Patients were stratified into three groups according to the changes in supine SBP during hemodialysis; $\mathrm{G}_{\mathrm{HTN}}$ (SBP increase $\geqslant 10 \mathrm{~mm} \mathrm{Hg}$ ), $\quad \mathrm{G}_{\mathrm{DROP}}<15 \mathrm{mmHg} \quad$ (SBP drop $<15 \mathrm{~mm} \mathrm{Hg}$ ) and $\mathrm{G}_{\text {DROP }} \geqslant 15 \mathrm{mmHg}$ (SBP drop $\geqslant 15 \mathrm{~mm} \mathrm{Hg}$ ). Two patients whose SBP increased $<10 \mathrm{~mm} \mathrm{Hg}$ during hemodialysis were excluded as our focus was to elucidate echocardiographic and hemodynamic characteristics in those with a significant increase in their SBP during hemodialysis. One patient was also excluded from the $\mathrm{G}_{\text {HTN }}$ group as they took etilefrine hydrochloride to elevate his SBP during hemodialysis. Written informed consent was obtained from all patients, and the protocol was approved by the Human Studies Subcommittee of Mie University Graduate School of Medicine.

\section{Hemodialytic procedure}

All patients underwent hemodialysis three times per week using either polysulfone $(n=63)$, polyester polymer alloy $(n=17)$, polymethyl methacrylate $(n=3)$ or cellulose triacetate $(n=1)$ dialyzers. Hemodialysis was performed for $3.5-5 \mathrm{~h}$ (average $3.8 \pm 0.6 \mathrm{~h}$ ) with acetate bicarbonate-buffered dialysate (sodium $138-143 \mathrm{mmoll}^{-1}$, potassium $2.0-2.1 \mathrm{mmoll}^{-1}$ and bicarbonate 24.0-29.4 $\mathrm{mmoll}^{-1}$ ). During each hemodialysis session, excess fluid was removed to achieve the patient's clinically determined dry weight, and the mean ultrafiltration volume was $1.8 \pm 0.81$. The median (interquartile range) vintage on hemodialysis was 54 (22-102) months.

\section{Study protocol}

All medications were continued during all testing sessions. After at least $20 \mathrm{~min}$ of supine rest, heart rate, SBP and diastolic BP were measured using an automated BP measuring device to establish pre-dialysis BPs and heart rate. BPs and heart rate were monitored hourly throughout hemodialysis and postdialysis measurements were performed in the supine position $15 \mathrm{~min}$ after disconnecting from the dialysis circuit.

\section{Echocardiography}

Two-dimensional Doppler echocardiography (HD11 XE; Philips Medical Systems, Bothell, WA, USA) was performed in the supine or slight left lateral decubitus position before hemodialysis and repeated at the end of hemodialysis by the same sonographer. LV posterior wall thickness at end-diastole, and LV end-diastolic and end-systolic dimensions were measured from the parasternal long-axis acoustic window. ${ }^{13}$

Peak early (E) and late (A) transmitral filling velocities were recorded, and the ratio of E/A was used to assess global LV diastolic filling. Peak early $\left(\mathrm{E}^{\prime}\right)$ and late $\left(\mathrm{A}^{\prime}\right)$ diastolic mitral annular velocities were measured at the septal side of the mitral annulus. ${ }^{14} \mathrm{E}^{\prime}$ was used to evaluate LV early diastolic filling including LV relaxation, ${ }^{14}$ and the ratio of peak early mitral inflow-to-peak early annular velocity ( $\mathrm{E} / \mathrm{E}^{\prime}$ ratio) was used to estimate LV end-diastolic pressure. ${ }^{15,16}$ All echocardiographic values represent the average of three cardiac cycles.

\section{Clinical outcomes}

After baseline measurements of hemodynamics and LV diastolic function, all patients were followed up for 4.5 years (range 7-54 months, average $41 \pm 17$ months). The study end-point was cardiovascular death or noncardiovascular death. The causes of death were determined by the attending doctors who were blinded to group assignment.

\section{Statistical analysis}

Statistical analyses were performed using SPSS 17.0 (Chicago, IL, USA). Data are expressed as mean \pm s.d. in tables and mean \pm s.e. in figures. Continuous data were compared by a one-way analysis of variance (ANOVA) with post-hoc analysis or nonparametric Kruskal-Wallis test depending on the outcome of tests for normality. Categorical data were assessed by chi-square test. A two-way repeated measures ANOVA was used to evaluate main (time; group) and interaction effects (time $\times$ group) on hemodynamic and echocardiographic variables before and after hemodialysis. Post-hoc analysis was used for pre-post comparisons where either the main or interaction effect was statistically significant. Simple and multivariate binary logistic analyses with forward stepwise procedure $(P<0.10$ for entry) were applied to assess the relationships between intradialytic-HTN and clinical variables. Event-free survival curves for cardiovascular or noncardiovascular deaths were constructed using Kaplan-Meier method in the three groups, and statistical differences among the three groups were assessed by the log-rank test. The impact of clinical predictors on cardiovascular events was assessed by simple and multivariate Cox regression analyses adjusted for age, gender, hemodialysis vintage and the presence of diabetes mellitus. A $P$-value $<0.05$ was considered significant.

\section{RESULTS}

\section{Patient characteristics}

As shown in Table $1, \mathrm{G}_{\mathrm{HTN}}$ had a higher male/female ratio compared with $\mathrm{G}_{\mathrm{DROP}}<15 \mathrm{mmHg}$ and $\mathrm{G}_{\mathrm{DROP}} \geqslant 15 \mathrm{mmHg}(P<0.05)$. Age, height, weight, pre-hemodialysis heart rate and medications were not significantly different among the three subsets of patients. Prehemodialysis SBP was significantly lower in $\mathrm{G}_{\mathrm{HTN}}(138 \pm 22 \mathrm{~mm} \mathrm{Hg})$ than in $\mathrm{G}_{\mathrm{DROP}}<15 \mathrm{~mm} \mathrm{Hg}(154 \pm 20 \mathrm{~mm} \mathrm{Hg})$ and $\mathrm{G}_{\mathrm{DROP}} \geqslant 15 \mathrm{~mm} \mathrm{Hg}$ $(165 \pm 17 \mathrm{~mm} \mathrm{Hg} ; \quad P \leqslant 0.01)$. Likewise, pre-hemodialysis diastolic BP was also lower in $\mathrm{G}_{\mathrm{HTN}}(75 \pm 11 \mathrm{~mm} \mathrm{Hg})$ compared with $\mathrm{G}_{\mathrm{DROP}} \geqslant 15 \mathrm{mmHg} \quad(85 \pm 9 \mathrm{mmHg}, P<0.05)$. Serum potassium was lower, whereas blood glucose was higher in $\mathrm{G}_{\mathrm{HTN}}$ compared with $\mathrm{G}_{\mathrm{DROP}} \geqslant 15 \mathrm{mmHg} \quad(P \leqslant 0.02)$. Interdialytic weight gain in $\mathrm{G}_{\mathrm{HTN}}$ $(1.2 \pm 0.6 \mathrm{~kg})$ was not different from $\mathrm{G}_{\mathrm{DROP}<15 \mathrm{~mm} \mathrm{Hg}}(1.7 \pm 0.8 \mathrm{~kg})$ or $\mathrm{G}_{\mathrm{DROP}}<15 \mathrm{~mm} \mathrm{Hg}(1.7 \pm 0.9 \mathrm{~kg}, P=0.07$; Table 2$)$.

\section{Hemodynamics during hemodialysis}

As shown in Figure 1a and Table 2, SBP continuously increased throughout hemodialysis in $\mathrm{G}_{\mathrm{HTN}}$, whereas it decreased in the other groups (interaction effect $P<0.01$ ). The average change in SBP was $29 \pm 12 \mathrm{~mm} \mathrm{Hg}$ in $\mathrm{G}_{\mathrm{HTN}},-7 \pm 4 \mathrm{~mm} \mathrm{Hg}$ in $\mathrm{G}_{\mathrm{DROP}}<15 \mathrm{~mm} \mathrm{Hg}$, and $-30 \pm 10 \mathrm{~mm} \mathrm{Hg}$ in $\mathrm{G}_{\mathrm{DROP}} \geqslant 15 \mathrm{~mm} \mathrm{Hg}$. As a result, post-hemodialysis SBP was significantly higher in $\mathrm{G}_{\mathrm{HTN}}(P<0.001)$. Similar to SBP, diastolic BP increased only in $\mathrm{G}_{\mathrm{HTN}}$ (interaction effect $P<0.01$ ). Heart rate deceased in all groups with hemodialysis (interaction effect $P=0.49 ;$ Figure 2 and Table 2).

\section{LV size and Doppler measures of diastolic function}

Before hemodialysis, $\mathrm{E}^{\prime}$ was lower in $\mathrm{G}_{\mathrm{HTN}}\left(4.0 \pm 1.2 \mathrm{~cm} \mathrm{~s}^{-1}\right)$ than in $\mathrm{G}_{\mathrm{DROP}}<15 \mathrm{~mm} \mathrm{Hg}\left(4.4 \pm 1.3 \mathrm{~cm} \mathrm{~s}^{-1}\right)$ or $\mathrm{G}_{\mathrm{DROP}} \geqslant 15 \mathrm{~mm} \mathrm{Hg}\left(4.9 \pm 1.9 \mathrm{~cm} \mathrm{~s}^{-1}\right)$, however, this effect did not reach conventional levels of statistical significance $(P=0.11)$. No significant pre-hemodialysis differences were observed in other echocardiographic parameters including $\mathrm{E} / \mathrm{E}^{\prime}$ ratio, LV end-diastolic dimension, LV end-systolic dimension or LV ejection fraction.

Hemodialysis decreased LV end-systolic dimension in all three groups $(P \leqslant 0.06)$ with no change in LV ejection fraction (Table 3$)$. 
Table 1 Patient characteristics

\begin{tabular}{|c|c|c|c|c|c|}
\hline & $A L L$ & $G_{\text {HTN }}$ & $G_{D R O P<15 \mathrm{~mm} \mathrm{Hg}}$ & $G_{D R O P} \geqslant 15 \mathrm{~mm} \mathrm{Hg}$ & ANOVA $\mathrm{P}$ \\
\hline Number & 84 & 21 & 32 & 31 & - \\
\hline Male gender, $n(\%)$ & $37(44)$ & $14(67)^{* \dagger}$ & $10(31)$ & $13(40)$ & 0.03 \\
\hline Age, years & $70 \pm 9$ & $70 \pm 9$ & $71 \pm 8$ & $68 \pm 2$ & 0.49 \\
\hline Height, cm & $155 \pm 9$ & $159 \pm 7$ & $154 \pm 10$ & $155 \pm 10$ & 0.14 \\
\hline Body weight, kg & $51.7 \pm 10.2$ & $51.2 \pm 7.0$ & $51.7 \pm 12.8$ & $52.0 \pm 9.2$ & 0.96 \\
\hline Body surface area, $\mathrm{m}^{2}$ & $1.47 \pm 0.17$ & $1.50 \pm 0.13$ & $1.46 \pm 0.20$ & $1.47 \pm 0.16$ & 0.97 \\
\hline $\mathrm{SBP}, \mathrm{mm} \mathrm{Hg}$ & $154 \pm 22$ & $138 \pm 22^{* \dagger}$ & $154 \pm 20$ & $165 \pm 17$ & $<0.01$ \\
\hline $\mathrm{DBP}, \mathrm{mm} \mathrm{Hg}$ & $81 \pm 12$ & $75 \pm 11^{\dagger}$ & $80 \pm 13$ & $85 \pm 9$ & $<0.01$ \\
\hline Heart rate, b.p.m. & $77 \pm 13$ & $76 \pm 13$ & $76 \pm 10$ & $78 \pm 15$ & 0.71 \\
\hline Cardiothoracic ratio, $n$ (\%) & $52 \pm 6$ & $50 \pm 4(45-58)$ & $54 \pm 6(45-77)$ & $52 \pm 6(38-62)$ & 0.10 \\
\hline \multicolumn{6}{|l|}{ Laboratory data } \\
\hline Serum albumin, $\mathrm{gdl}^{-1}$ & $3.6 \pm 0.4$ & $3.6 \pm 0.4$ & $3.6 \pm 0.3$ & $3.7 \pm 0.4$ & 0.55 \\
\hline Total cholesterol, $\mathrm{mgdl}^{-1}$ & $161 \pm 40$ & $132 \pm 31^{* \dagger}$ & $171 \pm 40$ & $170 \pm 38$ & $<0.01$ \\
\hline Serum calcium, mg dl ${ }^{-1}$ & $8.9 \pm 1.0$ & $8.5 \pm 0.6$ & $9.2 \pm 1.1$ & $9.0 \pm 0.9$ & 0.06 \\
\hline Serum phosphate, $\mathrm{mgdl}^{-1}$ & $5.4 \pm 1.4$ & $4.8 \pm 1.2$ & $5.6 \pm 1.4$ & $5.7 \pm 1.6$ & 0.05 \\
\hline Serum ALT, UI-1 & $11 \pm 7$ & $13 \pm 8$ & $10 \pm 7$ & $10 \pm 6$ & 0.54 \\
\hline Serum sodium, $\mathrm{mEq}^{-1}$ & $140 \pm 3$ & $140 \pm 3$ & $140 \pm 4$ & $140 \pm 3$ & 0.95 \\
\hline Serum potassium, $\mathrm{mEq} \mathrm{I}^{-1}$ & $4.6 \pm 0.7$ & $4.3 \pm 0.7^{\dagger}$ & $4.6 \pm 0.8$ & $4.8 \pm 0.6$ & 0.02 \\
\hline Hemoglobin, $\mathrm{gdl}^{-1}$ & $10.3 \pm 1.2$ & $9.7 \pm 1.0$ & $10.4 \pm 1.3$ & $10.5 \pm 1.2$ & 0.08 \\
\hline White blood cells, $10^{3} \mu \mathrm{I}^{-1}$ & $5.5 \pm 1.5$ & $4.9 \pm 1.5$ & $5.8 \pm 1.5$ & $5.6 \pm 1.4$ & 0.06 \\
\hline Platelet count, $10^{9} \mathrm{l}^{-1}$ & $173 \pm 52$ & $139 \pm 57^{* \dagger}$ & $190 \pm 43$ & $179 \pm 49$ & $<0.01$ \\
\hline Blood glucose, $\mathrm{mgdl}^{-1}$ & $136 \pm 52$ & $158 \pm 55^{\dagger}$ & $143 \pm 52$ & $114 \pm 43$ & 0.01 \\
\hline \multicolumn{6}{|l|}{ Comorbidities, $n(\%)$} \\
\hline Hypertension & $76(90)$ & $17(81)$ & $29(91)$ & $30(97)$ & 0.16 \\
\hline Dyslipidemia & $19(23)$ & $1(5)$ & $10(31)$ & $8(26)$ & 0.07 \\
\hline Diabetes mellitus & $39(46)$ & $13(62)$ & $16(50)$ & $10(32)$ & 0.10 \\
\hline Coronary artery disease & $10(12)$ & $5(24)$ & $2(6)$ & $3(10)$ & 0.14 \\
\hline Chronic glomerulonephritis & $30(35)$ & $6(29)$ & $8(25)$ & $15(16)$ & 0.40 \\
\hline \multicolumn{6}{|l|}{ Medication, $n(\%)$} \\
\hline ARB/ACE-inhibitors & $52(62)$ & $15(71)$ & $18(56)$ & $19(61)$ & 0.54 \\
\hline Calcium channel blockers & $43(51)$ & $13(62)$ & $16(50)$ & $14(45)$ & 0.30 \\
\hline Beta-blockers & $12(14)$ & $3(14)$ & $6(19)$ & $3(10)$ & 0.59 \\
\hline Insulin & $11(13)$ & $4(19)$ & $4(13)$ & $3(10)$ & 0.61 \\
\hline Oral antidiabetic drugs & $5(6)$ & $1(5)$ & $4(13)$ & $0(0)$ & 0.11 \\
\hline Antiphosphate drugs & $62(74)$ & $19(90)$ & $21(66)$ & $22(71)$ & 0.12 \\
\hline
\end{tabular}

Abbreviations: ACE, angiotensin-converting enzyme; ALT, alkaline phosphatase; ANOVA, analysis of variance; ARB, angiotensin II receptor antagonists; DBP, diastolic blood pressure;

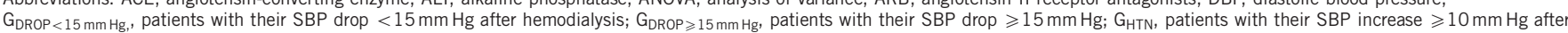
hemodialysis; SBP, systolic blood pressure.

Values are mean \pm s.d. or $n(\%)$. ${ }^{*} P<0.05$ vs. $\mathrm{G}_{\mathrm{DROP}}<15 \mathrm{~mm} \mathrm{Hg}$, and ${ }^{\dagger} P<0.05$ vs. $\mathrm{G}_{\mathrm{DROP}} \geqslant 15 \mathrm{~mm} \mathrm{Hg}$

The magnitude of $\mathrm{E}$ was significantly lower after hemodialysis in $\mathrm{G}_{\mathrm{DROP}}<15 \mathrm{mmHg}\left(74 \pm 19\right.$ vs. $\left.63 \pm 14 \mathrm{~cm} \mathrm{~s}^{-1}, \quad P=0.001\right)$ and $\mathrm{G}_{\mathrm{DROP}} \geqslant 15 \mathrm{~mm} \mathrm{Hg}\left(71 \pm 18\right.$ vs. $\left.58 \pm 14 \mathrm{~cm} \mathrm{~s}^{-1}, P<0.001\right)$, but remained unchanged in $\mathrm{G}_{\mathrm{HTN}}\left(70 \pm 21\right.$ vs. $\left.68 \pm 20 \mathrm{~cm} \mathrm{~s}^{-1}, P=0.50\right)$. $\mathrm{E}^{\prime}$ was lower after hemodialysis (time effect $P<0.001$ ), especially in $\mathrm{G}_{\mathrm{DROP}}<15 \mathrm{mmHg}$. After hemodialysis, $\mathrm{E} / \mathrm{E}^{\prime}$ ratio decreased only in $\mathrm{G}_{\mathrm{DROP}} \geqslant 15 \mathrm{mmHg}$. The ratio was larger in $\mathrm{G}_{\mathrm{HTN}}$ compared with $\mathrm{G}_{\mathrm{DROP}} \geqslant 15 \mathrm{mmHg}$ after hemodialysis $(20.0 \pm 7.0$ vs. $13.8 \pm 6.2$, $P=0.02$ ), suggesting a higher LV filling pressure in $\mathrm{G}_{\mathrm{HTN}}$ compared with $\mathrm{G}_{\mathrm{DROP}} \geqslant 15 \mathrm{~mm} \mathrm{Hg}$.

\section{Kaplan-Meier analysis}

During the follow-up period (average: $41 \pm 17$ months, range 7-54 months), all-cause deaths were observed in 11 out of 21 patients $(52 \%)$ in $\mathrm{G}_{\mathrm{HTN}}, 15$ out of $32(47 \%)$ in $\mathrm{G}_{\mathrm{DROP}}<15 \mathrm{~mm} \mathrm{Hg}$ and 13 out of $31(42 \%)$ in $G_{D R O P} \geqslant 15 \mathrm{~mm} \mathrm{Hg}$. No difference was observed in the rates of all-cause death among the groups (Figure 3). Cardiovascular deaths were observed in six patients in $\mathrm{G}_{\mathrm{HTN}}$ (29\%; two sudden deaths, two brain hemorrhage, one myocardial infarction and one ventricular arrhythmia), four patients in $\mathrm{G}_{\mathrm{DROP}} \geqslant 15 \mathrm{~mm} \mathrm{Hg}$ ( $13 \%$; one myocardial infarction, one aortic dissection, one heart failure and one acute peripheral arterial occlusion) and two patients (7\%; one stroke and one brain hemorrhage) in $\mathrm{G}_{\mathrm{DROP}} \geqslant 15 \mathrm{mmHg}$. As shown in Figure $4 \mathrm{a}$, $\mathrm{G}_{\mathrm{HTN}}$ had a higher risk of cardiovascular deaths (29\%) than $\mathrm{G}_{\mathrm{DROP}} \geqslant 15 \mathrm{mmHg} \quad(6 \%, P=0.03)$ and $\mathrm{G}_{\mathrm{DROP}<15 \mathrm{mmHg}} \quad(13 \%$, $P=0.12)$. Conversely, no difference was observed in the rate of noncardiovascular deaths among the groups $(P \geqslant 0.53)$ (Figure $4 b$ ). Infection was the leading cause of noncardiovascular deaths in all groups $\left(60 \%\right.$ in $\mathrm{G}_{\mathrm{HTN}}, 73 \%$ in $\mathrm{G}_{\mathrm{DROP}}<15 \mathrm{mmHg}$ and $45 \%$ in $\left.\mathrm{G}_{\mathrm{DROP}} \geqslant 15 \mathrm{~mm} \mathrm{Hg}\right)$.

Correlations between intradialytic-HTN and clinical variables Results of simple and multivariate logistic regression analyses evaluating clinical predictors of intradialytic-HTN are shown in 
Table 2 Hemodynamics during dialysis

\begin{tabular}{|c|c|c|c|c|}
\hline & $G_{H T N}$ & $G_{D R O P}<15 \mathrm{~mm} \mathrm{Hg}$ & $G_{D R O P} \geqslant 15 \mathrm{~mm} \mathrm{Hg}$ & ANOVA P \\
\hline Dialysis vintage, years & $5.6 \pm 7.3$ & $7.2 \pm 8.2$ & $6.8 \pm 6.4$ & 0.72 \\
\hline Ultrafiltration volume, kg & $1.8 \pm 0.8$ & $2.2 \pm 0.7$ & $2.1 \pm 0.8$ & 0.19 \\
\hline Filtration volume/hour, $\mathrm{kgh}^{-1}$ & $0.49 \pm 0.19$ & $0.58 \pm 0.18$ & $0.56 \pm 0.19$ & 0.21 \\
\hline Interdialytic weight gain, kg & $1.2 \pm 0.6$ & $1.7 \pm 0.8$ & $1.7 \pm 0.9$ & 0.07 \\
\hline Changes in SBP (pre-post), $\mathrm{mm} \mathrm{Hg}$ & $-29 \pm 12$ & $7 \pm 4$ & $29 \pm 10$ & $<0.01$ \\
\hline \multicolumn{5}{|l|}{ Supine SBP, $\mathrm{mm} \mathrm{Hg}$} \\
\hline Pre & $138 \pm 22$ & $154 \pm 20$ & $165 \pm 17$ & $<0.01$ \\
\hline $1 \mathrm{~h}$ & $149 \pm 20 *$ & $150 \pm 20$ & $147 \pm 17^{*}$ & \\
\hline $2 \mathrm{~h}$ & $157 \pm 22^{*}$ & $147 \pm 20^{*}$ & $143 \pm 17^{*}$ & \\
\hline $3 \mathrm{~h}$ & $162 \pm 24^{* \dagger}$ & $145 \pm 17^{*}$ & $134 \pm 15^{* * \ddagger}$ & \\
\hline Post & $168 \pm 21^{* * \neq}$ & $147 \pm 19 *$ & $135 \pm 14^{* * \ddagger}$ & \\
\hline \multicolumn{5}{|l|}{ Supine $D B P, \mathrm{~mm} \mathrm{Hg}$} \\
\hline Pre & $75 \pm 11$ & $80 \pm 13$ & $85 \pm 9$ & $<0.01$ \\
\hline $1 \mathrm{~h}$ & $77 \pm 8$ & $76 \pm 11$ & $78 \pm 7^{*}$ & \\
\hline $2 \mathrm{~h}$ & $81 \pm 5$ & $73 \pm 17^{*}$ & $76 \pm 9^{*}$ & \\
\hline $3 \mathrm{~h}$ & $82 \pm 10$ * & $74 \pm 8^{*}$ & $74 \pm 10^{*}$ & \\
\hline Post & $82 \pm 8^{*}$ & $76 \pm 11^{*}$ & $74 \pm 10^{*}$ & \\
\hline \multicolumn{5}{|l|}{ Supine heart rate, b.p.m. } \\
\hline Pre & $76 \pm 13$ & $76 \pm 11$ & $78 \pm 15$ & 0.71 \\
\hline $1 \mathrm{~h}$ & $72 \pm 10$ & $72 \pm 9^{*}$ & $70 \pm 10^{*}$ & \\
\hline $2 \mathrm{~h}$ & $71 \pm 5$ & $71 \pm 7^{*}$ & $73 \pm 9 *$ & \\
\hline $3 \mathrm{~h}$ & $71 \pm 7$ & $71 \pm 9$ & $74 \pm 8^{*}$ & \\
\hline Post & $72 \pm 7$ & $72 \pm 8$ & $74 \pm 8^{*}$ & \\
\hline
\end{tabular}

Abbreviations: ANOVA, analysis of variance; DBP, diastolic blood pressure; $G_{D R O P}<15 \mathrm{~mm} \mathrm{Hg}$, patients with their SBP drop $<15 \mathrm{~mm} \mathrm{Hg}$ after hemodialysis; $G_{D R O P} \geqslant 15 \mathrm{~mm} \mathrm{Hg}$, patients with their SBP drop $\geqslant 15 \mathrm{~mm} \mathrm{Hg}$; G hemodialysis; SBP, systolic blood pressure.

Data are expressed as mean \pm s.d. or $n(\%)$. ${ }^{*} P<0.05$ vs. pre-dialysis, ${ }^{\star} P<0.05$ vs. $1 \mathrm{~h}$ into dialysis, and $\ddagger P<0.05$ vs. $2 \mathrm{~h}$ into dialysis in each group.

Table 4. Multivariate regression analysis showed that blood glucose was positively correlated to intradialytic-HTN (odds ratio: 1.03, 95\% confidence interval (CI): 1.00-1.07), whereas cardiothoracic ratio (odds ratio: $0.67,95 \%$ CI: 0.51-0.89), SBP before hemodialysis (odds ratio: $0.87,95 \% \mathrm{CI}: 0.79-0.95)$, the change in $\mathrm{E} / \mathrm{E}^{\prime}$ ratio during hemodialysis (odds ratio: 0.74, 95\% CI: 0.59-0.92), and platelet count (odds ratio: $0.48,95 \% \mathrm{CI}: 0.29-0.80$ ) were negatively correlated to intradialytic-HTN. Serum phosphate and $\mathrm{E}^{\prime}$ before hemodialysis were negatively correlated to intradialytic-HTN, but neither of these effects reached conventional levels of statistical significance $(P=0.08$ and $P=0.07$, respectively).

Predictors of cardiovascular deaths

Results of simple and multivariate Cox proportional hazards model related to cardiovascular deaths are shown in Table 5. Previous coronary arterial disease and lower serum potassium were independently correlated to cardiovascular mortality; however, the relationship between intradialytic-HTN and cardiovascular mortality was not statistically significant. Dyslipidemia and diabetes mellitus appeared to be independently associated with cardiovascular deaths $(P=0.053$ and $P=0.06$, respectively).

\section{DISCUSSION}

In this study, we demonstrate that in patients with end-stage kidney disease intradialytic-HTN is associated with a smaller cardiothoracic ratio, a higher blood glucose level, and an increased LV afterload and filling pressure after hemodialysis. Intradialytic-HTN is also associated with an increased risk for adverse cardiovascular outcomes. a Systolic blood pressure

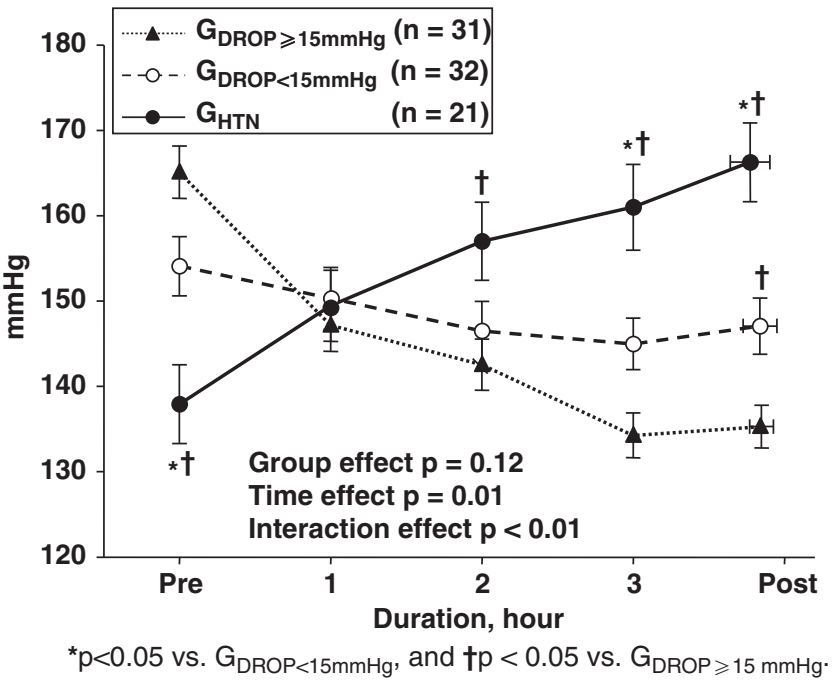

b

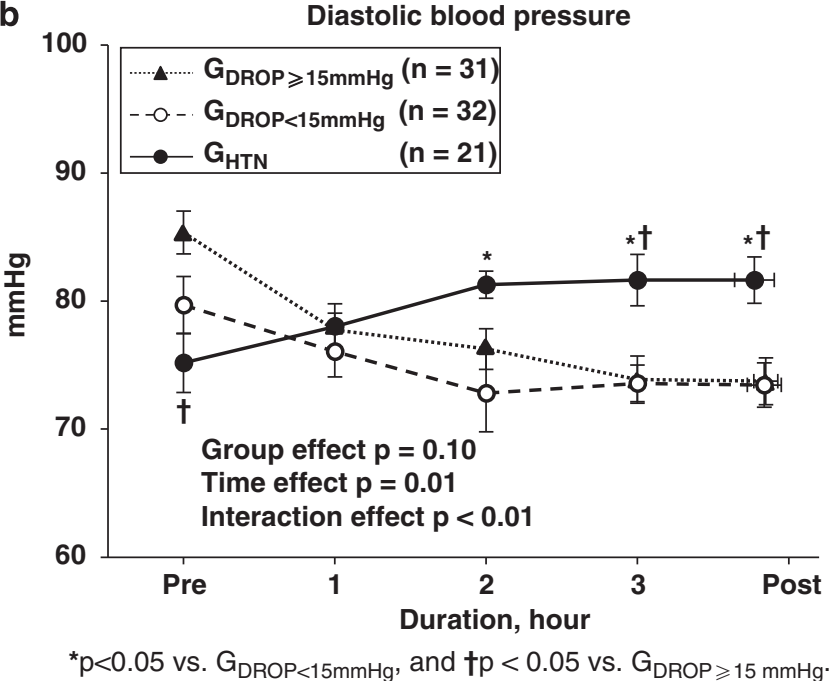

Figure 1 (a) Changes in systolic blood pressure (SBP) during hemodialysis. $\mathrm{G}_{\text {HTN }}$ indicates patients with their SBP increase $\geqslant 10 \mathrm{~mm} \mathrm{Hg}$ after hemodialysis; $G_{D R O P}<15 \mathrm{mmHg}$, SBP decrease $<15 \mathrm{~mm} \mathrm{Hg}$ after hemodialysis; $\mathrm{G}_{\mathrm{DROP}} \geqslant 15 \mathrm{~mm} \mathrm{Hg}$, SBP decrease $\geqslant 15 \mathrm{~mm} \mathrm{Hg}$ after hemodialysis. ${ }^{*} P<0.05$

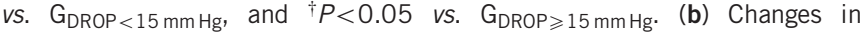
diastolic blood pressure during hemodialysis. ${ }^{*} P<0.05$ vs. $G_{D R O P}<15 \mathrm{~mm} \mathrm{Hg}$, and ${ }^{\dagger} P<0.05$ vs. G $\mathrm{DROP} \geqslant 15 \mathrm{~mm} \mathrm{Hg}$.

These findings suggest that increased cardiovascular stiffening and impaired LV diastolic filling may contribute in part to an increased cardiovascular burden in patients with intradialytic-HTN.

Hemodynamics in intradialytic-HTN during hemodialysis In the present study, $\sim 20 \%$ of patients were categorized as intradialytic-HTN using resting supine SBP, which is a slightly higher percentage than previously reported using upright seated SBP. ${ }^{10-12}$ Orthostatic stress decreases stroke volume and BP, resulting in an increased muscle sympathetic nerve activity. This sympathoexcitation effect to posture change may be particularly profound in the setting of dehydration or fluid loss. ${ }^{17}$ Thus, an increase in sympathetic nerve activity may also exist in intradialytic-HTN after hemodialysis in the present study. Our primary objective was to simultaneously evaluate 


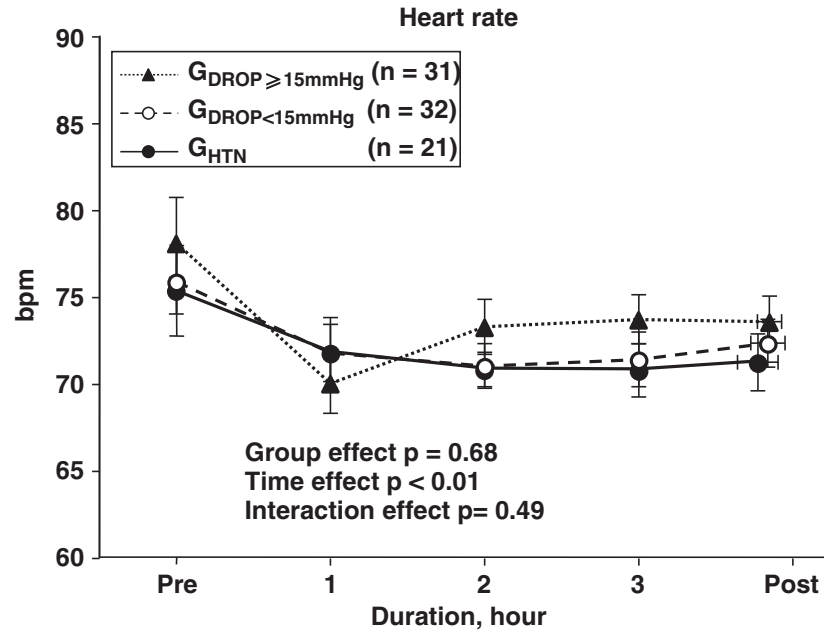

${ }^{*} p<0.05$ vs. $G_{D R O P}<15 \mathrm{mmHg}$, and $t p<0.05$ vs. $G_{D R O P} \geqslant 15 \mathrm{mmHg}$.

Figure 2 Changes in heart rate during hemodialysis. ${ }^{*} P<0.05$ vs

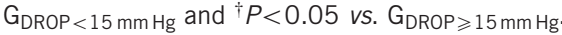

the impact of ultrafiltration on hemodynamics and echocardiographic parameters in our patients. Thus, to minimize the impact of systemic sympathoexcitation due to postural change, we used supine SBP to define intradialytic-HTN.

Although heart rate decreased during the early stages of hemodialysis in all groups, this reduction was blunted in $\mathrm{G}_{\mathrm{HTN}}$. In animals and humans, volume loading increases heart rate ${ }^{18}$ presumably due to the Bainbridge reflex mediated through inhibition of vagal outflow or enhanced sympathetic stimulation of the sinoatrial node by stretch receptors in the right and left atria. ${ }^{18}$ However, in an animal model of LV diastolic dysfunction and elevated filling pressures, a reduction in venous return 'unloads' these stretch receptors, resulting in a reflexinduced decrease in heart rate. ${ }^{19}$ Right atrial pressure may be higher in patients with a greater interdialytic weight gain before hemodialysis. Therefore, a larger volume of fluid needs to be removed by ultrafiltration to achieve the patient's dry weight. We speculate greater interdialytic weight gain in $\mathrm{G}_{\mathrm{DROP}}<15 \mathrm{~mm} \mathrm{Hg} \mathrm{mm} \mathrm{Hg}$ and $\mathrm{G}_{\mathrm{DROP}} \geqslant 15 \mathrm{mmHg}$ than in $\mathrm{G}_{\mathrm{HTN}}$ (ANOVA $P=0.07$ ) as shown in Table 2 might be related to the larger decrease in heart rate during hemodialysis in these two groups.

Finally, it has been demonstrated that the increase in total systemic vascular resistance is threefold greater during hemodialysis in patients with intradialytic-HTN compared with controls. ${ }^{20}$ The causes of this exaggerated response in patients with intradialytic-HTN are not completely clear, however, an imbalanced relationship between nitric oxide and endothelium expression is believed to contribute. ${ }^{20}$ Nevertheless, this elevated systemic vascular resistance in combination with increased cardiovascular stiffness and impaired LV diastolic function would increase cardiovascular burden in patients with intradialytic-HTN, and consequently may contribute to a greater risk of cardiovascular mortality.

\section{Echocardiographic characteristics in intradialytic-HTN}

Patients on hemodialysis are characterized by increased cardiovascular stiffening and severely impaired LV diastolic function. ${ }^{7}$ Given the clinical impact of intradialytic-HTN, it is surprising that no previous studies have evaluated LV diastolic function in patients with intradialytic-HTN. Intriguingly, hemodialysis did not affect $\mathrm{E}, \mathrm{E}^{\prime}$ or $\mathrm{E} / \mathrm{E}^{\prime}$ ratio in our $\mathrm{G}_{\mathrm{HTN}}$ patients. Increased $\mathrm{LV}$ afterload prolongs $\mathrm{LV}$
Table 3 Doppler echocardiography

\begin{tabular}{|c|c|c|c|c|c|c|}
\hline & $G_{\text {HTN }}$ & $G_{D R O P<15 \mathrm{~mm} \mathrm{Hg}}$ & $G_{D R O P} \geqslant 15 \mathrm{~mm} \mathrm{Hg}$ & $\begin{array}{l}\text { Group } \\
\text { effect } \mathrm{P}\end{array}$ & $\begin{array}{l}\text { Time } \\
\text { effect } \mathrm{P}\end{array}$ & $\begin{array}{c}\text { Interaction } \\
\text { effect } \mathrm{P}\end{array}$ \\
\hline N & 21 & 32 & 31 & & & \\
\hline \multicolumn{7}{|c|}{$L V D D, m m$} \\
\hline Pre & $43 \pm 7$ & $42 \pm 6$ & $41 \pm 7$ & 0.30 & $<0.001$ & 0.95 \\
\hline Post & $41 \pm 9$ & $40 \pm 7^{*}$ & $38 \pm 7^{*}$ & & & \\
\hline \multicolumn{7}{|c|}{ LVDS, $m m$} \\
\hline Pre & $27 \pm 6$ & $27 \pm 7$ & $27 \pm 6$ & 0.51 & 0.02 & 0.62 \\
\hline Post & $27 \pm 8$ & $25 \pm 6^{*}$ & $25 \pm 8$ & & & \\
\hline \multicolumn{7}{|c|}{ LV ejection fraction, $n(\%)$} \\
\hline Pre & $64 \pm 10$ & $65 \pm 10$ & $62 \pm 11$ & 0.67 & 0.57 & 0.93 \\
\hline Post & $63 \pm 13$ & $65 \pm 11$ & $63 \pm 14$ & & & \\
\hline \multicolumn{7}{|c|}{$E, c m s^{-1}$} \\
\hline Pre & $70 \pm 21$ & $74 \pm 19$ & $71 \pm 18$ & 0.41 & $<0.001$ & 0.11 \\
\hline Post & $68 \pm 20$ & $63 \pm 14^{*}$ & $58 \pm 14^{*}$ & & & \\
\hline \multicolumn{7}{|c|}{$A, \mathrm{cms}^{-1}$} \\
\hline pre & $92 \pm 24$ & $101 \pm 21$ & $90 \pm 21$ & 0.25 & 0.002 & 0.29 \\
\hline post & $89 \pm 19$ & $89 \pm 21^{*}$ & $84 \pm 23$ & & & \\
\hline \multicolumn{7}{|c|}{ Mitral E/A ratio } \\
\hline pre & $0.81 \pm 0.31$ & $0.74 \pm 0.15$ & $0.82 \pm 0.25$ & 0.49 & 0.07 & 0.24 \\
\hline post & $0.78 \pm 0.24$ & $0.72 \pm 0.17$ & $0.71 \pm 0.18$ & & & \\
\hline \multicolumn{7}{|c|}{$E, \mathrm{cms}^{-1}$} \\
\hline pre & $4.0 \pm 1.2$ & $4.4 \pm 1.3$ & $4.9 \pm 1.9$ & 0.02 & 0.02 & 0.64 \\
\hline post & $3.6 \pm 1.1^{\dagger}$ & $3.9 \pm 0.9^{*}$ & $4.7 \pm 1.6$ & & & \\
\hline \multicolumn{7}{|c|}{$A^{\prime}, \mathrm{cms}^{-1}$} \\
\hline Pre & $7.5 \pm 1.2$ & $7.6 \pm 1.9$ & $7.2 \pm 1.8$ & 0.66 & 0.89 & 0.65 \\
\hline Post & $7.7 \pm 2.8$ & $7.2 \pm 1.8$ & $7.1 \pm 2.3$ & & & \\
\hline \multicolumn{7}{|c|}{ E/E' ratio } \\
\hline Pre & $18.9 \pm 6.9$ & $18.4 \pm 7.5$ & $16.5 \pm 7.2$ & 0.03 & 0.13 & 0.10 \\
\hline Post & $20.0 \pm 7.0^{\dagger}$ & $16.7 \pm 4.4$ & $13.8 \pm 6.2^{*}$ & & & \\
\hline \multicolumn{7}{|c|}{ LVPWT, mm } \\
\hline Pre & $14 \pm 3$ & $14 \pm 5$ & $13 \pm 3$ & 0.71 & 0.37 & 0.70 \\
\hline Post & $13 \pm 3$ & $13 \pm 4$ & $13 \pm 3$ & & & \\
\hline
\end{tabular}

Abbreviations: $A$, peak late mitral inflow velocity; $A^{\prime}$, peak late mitral annular velocity; $E$, peak early mitral inflow velocity; $E^{\prime}$, peak early mitral annular velocity; $E / E^{\prime}$ ratio, the ratio of peak early mitral filling velocity-to-peak early mitral annular velocity; LVDD, left ventricular enddiastolic diameter; LVDS, left ventricular end-systolic diameter; LVPWT, left ventricular posterior wall thickness; SBP indicates systolic blood pressure.

Values are mean \pm s.d. ${ }^{*} P<0.05$ vs. pre in each group, and ${ }^{\dagger} P<0.05$ vs. $\mathrm{G}_{\mathrm{DROP}} \geqslant 15 \mathrm{~mm} \mathrm{Hg}$.

relaxation and may shift LV pressure-volume relationship upward, thereby increasing LV filling pressure. ${ }^{21}$ In the present study, LV filling pressure estimated by $\mathrm{E} / \mathrm{E}^{\prime}$ ratio was unaffected after hemodialysis in $\mathrm{G}_{\mathrm{HTN}}$. We speculate that the effect of preload reduction by ultrafiltration on LV filling pressure was counterbalanced by that of increased LV afterload in $\mathrm{G}_{\mathrm{HTN}}$, and that elevated LV filing pressure in $\mathrm{G}_{\mathrm{HTN}}$ after hemodialysis may in part contribute to an increased risk of cardiovascular mortality in $\mathrm{G}_{\mathrm{HTN}}$.

\section{Predictors for intradialytic-HTN}

The pathogenesis of intradialytic-HTN is proposed to be multifactorial. ${ }^{22}$ For example, Yang et al. ${ }^{12}$ reported that intradialytic-HTN was independently associated with volume overload as observed in 
patients with a larger cardiothoracic ratio. Volume reduction protocols such as ultrafiltration or lower body negative pressure lower right and left ventricular end-diastolic volume, and decrease external constraint on the LV, especially in patients with volume overload. ${ }^{23,24}$ This decreased constraint on the LV may increase stroke volume and cardiac output ${ }^{25}$ and eventually elevate BP during hemodialysis. Therefore, in some patients with volume overload, an increase in SBP during the first few hours into hemodialysis was observed, which was followed by a gradual decrease in SBP. ${ }^{26}$ Contrary to the previous report, ${ }^{12}$ cardiothoracic ratio was inversely associated with intradialytic-HTN in the present study. We observed that supine SBP continued to elevate throughout hemodialysis in $\mathrm{G}_{\mathrm{HTN}}$. This finding may suggest that volume overload is not always associated with intradialytic-HTN.

We observed that high blood glucose was independently associated with intradialytic-HTN. Exposure to high blood glucose induces endothelial dysfunction ${ }^{27}$ and LV diastolic dysfunction, ${ }^{28}$ both of which are predictors of intradialytic-HTN. ${ }^{22}$ Higher blood glucose in

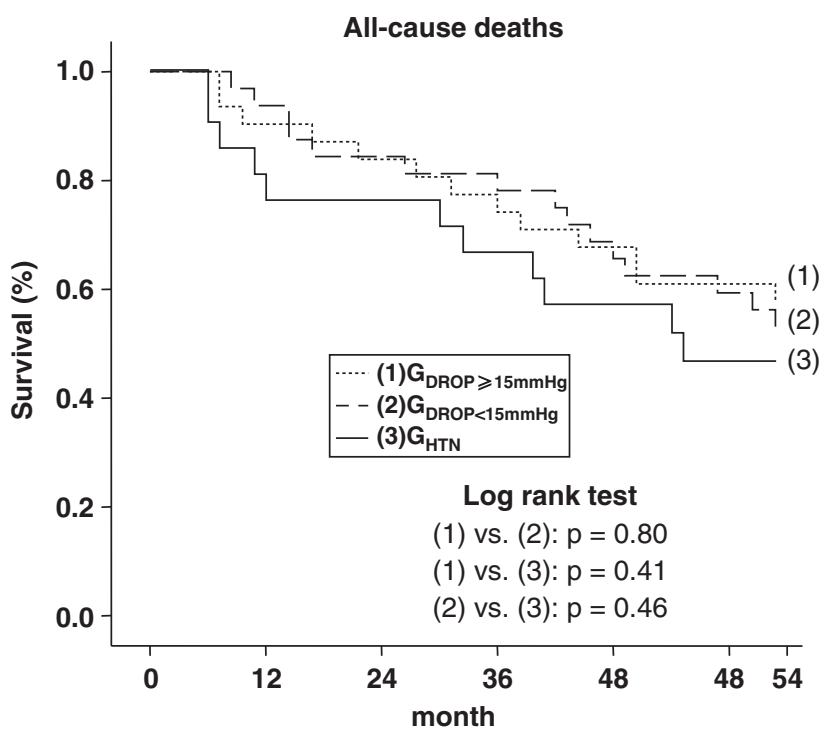

Figure 3 Kaplan-Meier analysis showing all-cause mortality rate in $\mathrm{G}_{\text {HTN }}$, $G_{D R O P}<15 \mathrm{mmHg}$, and $G_{D R O P} \geqslant 15 \mathrm{mmHg}$. No difference was observed in allcause mortality rate among the three groups.

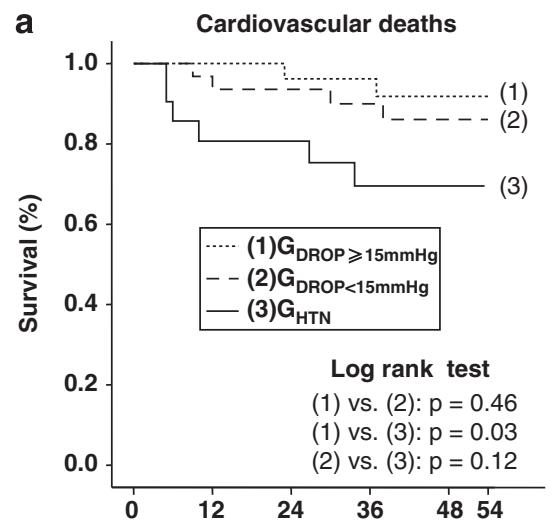

$\mathrm{G}_{\text {HTN }}$ might also reflect greater impairments of endothelial function. Exaggerated sympathetic nerve activity has been reported to be another predictor of intradialytic-HTN. ${ }^{22,29}$ Unfortunately, we did not measure catecholamine levels before and after hemodialysis in the present study. However, ultrafiltration would decrease LV stroke volume and induce an overstimulation of sympathetic nerve system in $\mathrm{G}_{\mathrm{HTN}}$ who may not be severely volume-overloaded. We observed that platelet count was inversely correlated to intradialytic-HTN. This result remains unexplained and may need to be examined in a larger population.

\section{Cardiovascular mortality in patients on hemodialysis}

In the present study, serum potassium (odds ratio $=0.31, P=0.01$ ) as well as previous coronary artery disease were independently associated with cardiovascular death. Hyperkalemia and hypokalemia have been reported to be associated with increased mortality in patients on regular hemodialysis. ${ }^{30-32}$ Kovesdy et al. $^{33}$ reported that a serum potassium level between 4.6 and $5.3 \mathrm{mEql}^{-1}$ resulted in the greatest survival, whereas levels $<4.0$ or $\geqslant 5.6 \mathrm{mEql}^{-1}$ were associated with increased mortality. In the present study, 16 patients out of $84(19 \%)$ had pre-hemodialysis potassium $<4.0 \mathrm{mEql}^{-1}$, whereas only $7(8 \%)$ had pre-hemodialysis potassium $\geqslant 5.6 \mathrm{mEql}^{-1}$. No patients in $\mathrm{G}_{\mathrm{HTN}}$ had pre-hemodialysis serum potassium $>5.0 \mathrm{mEql}^{-1}$. In addition, patients in $\mathrm{G}_{\mathrm{HTN}}$ appeared to have lower total cholesterol and serum phosphate, and less interdialytic weight gain (ANOVA, $P \leqslant 0.07$ ) compared with the other groups. These findings may suggest a possible malnutrition state in intradialytic-HTN.

Recently, Yang et al. ${ }^{12}$ reported that intradialytic-HTN was associated with high cardiothoracic ratio, and that intradialyticHTN was an independent predictor for all-cause mortality over a 4-year follow-up period. Contrary to this previous work, we did not find any significant association between intradialytic-HTN and cardiovascular death or all-cause mortality over a similar follow-up period. This discrepancy between studies might be partly explained by differences in patient's characteristics including volume status in the two studies. It is possible that some patients with intradialytic-HTN in the previous study of Yang et al. ${ }^{12}$ may have had greater LV external constraint caused by volume overload before hemodialysis, and therefore may explain the positive relationship between intradialytic-HTN and cardiovascular death reported by these authors.

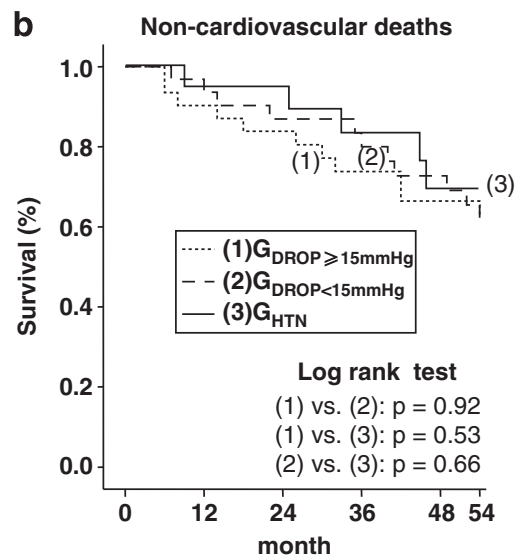

Figure 4 (a) Kaplan-Meier analysis showing cardiovascular mortality rate in $G_{H T N}, G_{D R O P}<15 \mathrm{mmHg}$ and $G_{D R O P} \geqslant 15 \mathrm{~mm} H$. A higher cardiovascular mortality rate was observed in $\mathrm{G}_{H T N}$ compared to $\mathrm{G}_{\mathrm{DROP}} \geqslant 15 \mathrm{mmHg}(P=0.03)$. (b) Kaplan-Meier analysis showing noncardiovascular mortality rate in $\mathrm{G}_{H T N}$, $G_{D R O P}<15 \mathrm{~mm} \mathrm{Hg}$ and $G_{D R O P} \geqslant 15 \mathrm{~mm} \mathrm{Hg}$. No difference was observed in noncardiovascular mortality rate among the three groups. 
Table 4 Logistic regression analysis of the factors for intradialyticHTN

\begin{tabular}{|c|c|c|c|c|}
\hline & \multicolumn{2}{|r|}{ Univariate } & \multicolumn{2}{|r|}{ Multivariate } \\
\hline & P-value & Odds ratio & P-value & Odds ratio \\
\hline Age, 10 year & 0.72 & $1.11(0.63-1.95)$ & & \\
\hline Male gender & 0.01 & $3.73(1.31-10.6)$ & 0.95 & \\
\hline Body surface area, $\mathrm{m}^{2}$ & 0.43 & $0.32(0.18-58.6)$ & & \\
\hline Dialysis vintage, years & 0.43 & $0.97(0.90-1.05)$ & & \\
\hline Filtration volume/hour, $\mathrm{kg} \mathrm{h}^{-1}$ & 0.09 & $1.00(0.99-1.00)$ & 0.34 & \\
\hline Interdialytic weight gain, kg & 0.03 & $0.40(0.18-0.90)$ & 0.10 & \\
\hline $\mathrm{Kt} / \mathrm{v}$ & 0.27 & $0.62(0.25-1.48)$ & & \\
\hline Cardiothoracic ratio, $n(\%)$ & 0.06 & $0.90(0.81-1.00)$ & 0.005 & $0.67(0.51-0.89)$ \\
\hline Supine SBP pre, mm Hg & $<0.001$ & $0.94(0.91-0.97)$ & 0.003 & $0.87(0.79-0.95)$ \\
\hline Supine DBP pre, mm Hg & 0.01 & $0.94(0.90-0.99)$ & 0.21 & \\
\hline Supine heart rate pre, b.p.m. & 0.65 & $0.99(0.95-1.03)$ & & \\
\hline$\Delta$ Heart rate (pre-post), b.p.m. & 0.92 & $1.00(0.96-1.05)$ & & \\
\hline \multicolumn{5}{|l|}{ Echocardiographic variables } \\
\hline LVDD pre, mm & 0.47 & $1.03(0.95-1.11)$ & & \\
\hline LVEF pre, \% & 0.96 & $1.12(0.01-134.6)$ & & \\
\hline E pre, $\mathrm{cms}^{-1}$ & 0.67 & $0.99(0.97-1.02)$ & & \\
\hline Mitral E/A ratio pre & 0.60 & $1.73(0.23-13.2)$ & & \\
\hline $\mathrm{E}^{\prime}$ pre, $\mathrm{cms}^{-1}$ & 0.09 & $0.72(0.49-1.06)$ & 0.07 & $0.37(0.13-1.07)$ \\
\hline $\mathrm{E} / \mathrm{E}^{\prime}$ ratio pre & 0.43 & $1.03(0.96-1.10)$ & & \\
\hline$\Delta \mathrm{LVDD}$ (pre-post), mm & 0.76 & 0.99 (0.89-1.09) & & \\
\hline$\Delta$ LVEF (pre-post), \% & 0.96 & $1.10(0.02-53.0)$ & & \\
\hline$\Delta \mathrm{E}$ (pre-post), $\mathrm{cms}^{-1}$ & 0.048 & $0.97(0.95-1.00)$ & 0.94 & \\
\hline$\Delta \mathrm{E} / \mathrm{A}$ ratio (pre-post) & 0.63 & $0.59(0.07-4.97)$ & & \\
\hline$\Delta \mathrm{E}^{\prime}$ (pre-post), $\mathrm{cm} \mathrm{s}^{-1}$ & 0.91 & $1.02(0.69-1.52)$ & & \\
\hline$\Delta \mathrm{E} / \mathrm{E}^{\prime}$ ratio (pre-post) & 0.047 & $0.92(0.84-0.99)$ & 0.007 & $0.74(0.59-0.92)$ \\
\hline \multicolumn{5}{|l|}{ Laboratory data } \\
\hline Serum albumin, $\mathrm{g} \mathrm{dl}^{-1}$ & 0.28 & $0.49(0.13-1.81)$ & & \\
\hline Total cholesterol, $\mathrm{mg} \mathrm{dl}^{-1}$ & 0.001 & $0.97(0.95-0.99)$ & 0.50 & \\
\hline Serum calcium, $\mathrm{mgdl}^{-1}$ & 0.03 & $0.48(0.25-0.92)$ & 0.10 & \\
\hline Serum phosphate, $\mathrm{mgdl}^{-1}$ & 0.02 & $0.61(0.40-0.93)$ & 0.08 & \\
\hline Serum ALT, U I-1 & 0.29 & $1.04(0.97-1.11)$ & & \\
\hline Serum sodium, $\mathrm{mEq} \mathrm{I}^{-1}$ & 0.83 & $1.02(0.87-1.19)$ & & \\
\hline Serum potassium, $\mathrm{mEq} \mathrm{I}^{-1}$ & 0.01 & $0.35(0.15-0.80)$ & 0.40 & \\
\hline Hemoglobin, $\mathrm{gdl}^{-1}$ & 0.04 & $0.62(0.39-0.97)$ & 0.36 & \\
\hline White blood cells, $10^{3} \mu \mathrm{l}^{-1}$ & 0.02 & $0.96(0.92-0.99)$ & 0.37 & \\
\hline Platelet count, $10^{9} \mathrm{I}^{-1}$ & 0.002 & $0.82(0.73-0.93)$ & 0.004 & $0.48(0.29-0.80)$ \\
\hline Blood glucose, mg dl-1 & 0.03 & $1.01(1.001-1.02)$ & 0.03 & 1.03 (1.003-1.07) \\
\hline \multicolumn{5}{|l|}{ Comorbidities, $n$ (\%) } \\
\hline Diabetes mellitus & 0.11 & $2.31(0.84-6.37)$ & & \\
\hline Hypertension & 0.10 & $0.29(0.07-1.28)$ & & \\
\hline Dyslipidemia & 0.05 & $0.13(0.02-1.00)$ & 0.10 & \\
\hline Coronary artery disease & 0.06 & $3.63(0.93-14.1)$ & 0.40 & \\
\hline \multicolumn{5}{|l|}{ Medication, $n(\%)$} \\
\hline ARB/ACE-inhibitors & 0.30 & $1.76(0.60-5.13)$ & & \\
\hline Calcium channel blockers & 0.14 & $2.20(0.78-6.18)$ & & \\
\hline Beta-blockers & 1.00 & $1.00(0.24-4.10)$ & & \\
\hline
\end{tabular}

Abbreviations: $\Delta$, changes in variables between pre- and post-dialysis; $A$, peak late mitral inflow velocity; ACE, angiotensin-converting enzyme; ALT, alkaline phosphatase; ARB, angiotensin II receptor antagonists; DBP, diastolic blood pressure; $E$, peak early mitral inflow velocity; $E^{\prime}$ peak early mitral annular velocity; Kt/v, dialysis adequacy; LVDD, left ventricular diastolic dimension. LVEF, left ventricular ejection fraction: SBP, systolic blood pressure.

\section{Study limitations}

There are several limitations to the current study. First, contrary to the previous studies using the upright position to determine intradialytic-HTN, ${ }^{10,34}$ we evaluated echocardiographic parameters
Table 5 Cox regression analysis for cardiovascular mortality in patients with dialysis

\begin{tabular}{|c|c|c|c|c|}
\hline & \multicolumn{2}{|c|}{ Simple regression } & \multicolumn{2}{|c|}{ Multiple regression } \\
\hline & P-value & Odds ratio & P-value & Odds ratio \\
\hline Age, 10 year & 0.73 & $1.16(0.51-2.64)$ & & \\
\hline Male gender & 0.32 & $1.87(0.55-6.40)$ & & \\
\hline Body surface area, $\mathrm{m}^{2}$ & 0.46 & $0.15(0.01-24.3)$ & & \\
\hline Dialysis vintage, years & 0.07 & $1.09(0.99-1.20)$ & 0.88 & \\
\hline Intradialytic HTN & 0.09 & $2.79(0.84-9.11)$ & 0.76 & \\
\hline Filtration volume/hour, $\mathrm{kgh}^{-1}$ & 0.89 & $1.00(0.99-1.00)$ & & \\
\hline Interdialytic weight gain, kg & 0.87 & $0.95(0.50-1.78)$ & & \\
\hline $\mathrm{Kt} / \mathrm{v}$ & 0.43 & $1.50(0.55-4.10)$ & & \\
\hline Cardiothoracic ratio, \% & 0.27 & $1.08(0.94-1.24)$ & & \\
\hline Supine SBP pre, mm Hg & 0.88 & $1.00(0.98-1.03)$ & & \\
\hline Supine SBP post, mm Hg & 0.06 & 1.02 (0.99-1.05) & 0.26 & \\
\hline
\end{tabular}

Echocardiographic variables

Baseline LVDD pre, mm $\quad 0.11 \quad 1.08(0.98-1.20)$

Baseline LVEF, \% $\quad 0.57 \quad 0.17(0.01-80.1)$

Baseline E wave, $\mathrm{cms}^{-1} \quad 0.74 \quad 1.00(0.97-1.03)$

Baseline mitral E/A ratio $\quad 0.65 \quad 0.55(0.04-7.30)$

Baseline $\mathrm{E}^{\prime}, \mathrm{cm} \mathrm{s}^{-1} \quad 0.63 \quad 0.87(0.49-1.54)$

Baseline E/E' $\quad 0.96 \quad 1.00(0.92-1.09)$

$\triangle$ LVDD (pre-post), $\mathrm{cm} \mathrm{s}^{-1} \quad 0.89 \quad 0.99(0.88-1.12)$

$\Delta \mathrm{LVEF}$ (pre-post), \% $\quad 0.74 \quad 2.20(0.02-215.9)$

$\Delta \mathrm{E}$ wave (pre-post), $\mathrm{cm} \mathrm{s}^{-1} \quad 0.27 \quad 1.02(0.98-1.06)$

$\Delta$ Mitral E/A (pre-post) $\quad 0.65 \quad 2.04(0.10-43.5)$

$\Delta \mathrm{E}^{\prime}$ (pre-post), $\mathrm{cm} \mathrm{s}^{-1} \quad 0.33 \quad 0.77(0.46-1.31)$

$\begin{array}{lll}\Delta \mathrm{E}^{\prime} \mathrm{E}^{\prime} \text { (pre-post) } & 0.12 & 0.92(0.83-1.02)\end{array}$

\section{Laboratory data}

$\begin{array}{llll}\text { Serum albumin, } \mathrm{g} \mathrm{dl}^{-1} & 0.04 & 14.9(1.10-200.3) & 0.07\end{array}$

Total cholesterol, $\mathrm{mg} \mathrm{dl}^{-1} \quad 0.30 \quad 1.01(0.99-1.03)$

Serum calcium, $\mathrm{mg} \mathrm{dl}^{-1} \quad 0.17 \quad 1.53(0.83-2.81)$

Serum phosphate, $\mathrm{mgdl}^{-1} \quad 0.62 \quad 0.88(0.54-1.45)$

Serum ALT, UI ${ }^{-1} \quad 0.38 \quad 1.03(0.96-1.11)$

Serum sodium, $\mathrm{mEqI}^{-1} \quad 0.81 \quad 1.02(0.85-1.24)$

Serum potassium, $\mathrm{mEqI}^{-1} \quad 0.04 \quad 0.41(0.18-0.97)$

Hemoglobin, $\mathrm{g} \mathrm{dl}^{-1} \quad 0.82 \quad 1.06(0.65-1.72)$

White blood cells, $10^{3} \mu \mathrm{l}^{-1} \quad 0.02 \quad 0.94(0.89-0.99)$

Platelet count, $10^{9} \mathrm{I}^{-1} \quad 0.21 \quad 0.94(0.84-1.04)$

Blood glucose, $\mathrm{mg} \mathrm{dl}^{-1} \quad 0.60 \quad 1.00(0.98-1.01)$

$0.01 \quad 0.31(0.12-0.77)$

0.06

Comorbidities, $n(\%)$

Diabetes mellitus

Hypertension

Dyslipidemia

Coronary artery disease

$\begin{array}{lll}0.02 & 9.97(1.57-63.4) & 0.06 \\ 0.86 & 0.83(0.54-6.39) & \\ 0.03 & 5.62(1.17-27.1) & 0.053 \\ 0.07 & 3.29(0.90-12.0) & 0.04\end{array}$

.06

$0.04 \quad 4.86(1.05-22.4)$

Medication, $n(\%)$

$\begin{array}{lll}\text { ARB/ACE-inhibitors } & 0.11 & 7.12(0.65-78.5) \\ \text { Calcium channel blockers } & 0.32 & 2.03(0.50-8.16) \\ \text { Beta-blockers } & 0.89 & 0.89(0.18-4.37)\end{array}$

Abbreviations: $\triangle$, changes in variables between pre and post dialysis: $A$, peak late mitral inflow velocity; ACE, angiotensin-converting enzyme; ALT, alkaline phosphatase; ARB, angiotensin II receptor antagonists; $E$ wave velocity, early diastolic transmitral velocity; $E^{\prime}$, peak early velocity of septal mitral annulus; $\mathrm{Kt} / \mathrm{v}$, dialysis adequacy; LVDD, left ventricular diastolic dimension; LVEF, left ventricular ejection fraction; SBP, systolic blood pressure.

Age, gender, dialysis vintage and the presence of diabetes mellitus were adjusted.

in the supine or slight left lateral decubitus position, and determined intradialytic-HTN by the use of supine SBP. These positions are the standardized postures for assessing LV geometry and function by echocardiography. We believe that the current protocol reduces the 
confounding effect of sympathetically mediated changes in hemodynamics in response to postural change. Second, the number of the patients was small for this type of analysis in the present study. In addition, the number of deaths from cardiovascular causes was very small, especially in $\mathrm{G}_{\mathrm{DROP}}<15 \mathrm{mmHg} \quad(n=2)$ and in $\mathrm{G}_{\text {DROP }} \geqslant 15 \mathrm{mmHg} \quad(n=1)$. Owing to the small sample size, our observations need to be examined in a larger sample size and the relationship between intradialytic-HTN and cardiovascular mortality should be evaluated in patients with no excessive volume overload before hemodialysis. Third, the number of the patients with previously diagnosed coronary artery disease was relatively small although patients on regular hemodialysis have a high risk for coronary artery disease. ${ }^{35}$ Although it is plausible that some of our patients had asymptomatic coronary artery disease, ${ }^{35}$ our results may not be applicable to a cohort in which the prevalence of coronary artery disease is high. Finally, the usage of a noninvasive measure of LV filling pressure could be considered a limitation of the current study. A couple of previous studies ${ }^{15,36}$ have reported that $\mathrm{E} / \mathrm{E}^{\prime}$ ratio provides a reasonable indication of $\mathrm{LV}$ filling pressure in patients; however, the precision of this relationship may weaken when evaluated on an individual basis or during significant changes in preload. ${ }^{37}$ Therefore, some caution is required for the current findings related to LV filling pressure in these patients.

\section{CONCLUSIONS}

Intradialytic-HTN was associated with a smaller cardiothoracic ratio and higher blood glucose in patients with end-stage renal disease on regular hemodialysis. Increased LV afterload and elevated LV filling pressure, indicative of increased cardiovascular stiffness and impaired LV diastolic filling, were observed after hemodialysis in patients with intradialytic-HTN, which may contribute at least in part to an increased risk for cardiovascular mortality.

\section{CONFLICT OF INTEREST}

The authors declare no conflict of interest.

\section{ACKNOWLEDGEMENTS}

We thank the staff of the hemodialysis unit for technical assistance. We also thank Dr Tomohiro Murata for valuable comments.

1 Agarwal R, Nissenson AR, Batlle D, Coyne DW, Trout JR, Warnock DG. Prevalence, treatment, and control of hypertension in chronic hemodialysis patients in the United States. Am J Med 2003; 115: 291-297.

2 Kuwahara F, Kai H, Tokuda K, Takeya M, Takeshita A, Egashira K, Imaizumi T. Hypertensive myocardial fibrosis and diastolic dysfunction: another model of inflammation? Hypertension 2004; 43: 739-745.

3 Weber KT, Janicki JS, Shroff SG, Pick R, Chen RM, Bashey RI. Collagen remodeling of the pressure-overloaded, hypertrophied nonhuman primate myocardium. Circ Res 1988; 62: 757-765.

4 Oki T, Tabata T, Yamada H, Wakatsuki T, Mishiro Y, Abe M, Onose Y, Iuchi A, Ito S. Left ventricular diastolic properties of hypertensive patients measured by pulsed tissue Doppler imaging. J Am Soc Echocardiogr 1998; 11: 1106-1112.

5 Franco $\mathrm{OH}$, Peeters A, Bonneux L, de Laet $\mathrm{C}$. Blood pressure in adulthood and life expectancy with cardiovascular disease in men and women: life course analysis. Hypertension 2005; 46: 280-286.

6 Agarwal R. The controversies of diagnosing and treating hypertension among hemodialysis patients. Semin Dial 2012; 25: 370-376.

7 Foley RN, Collins AJ. End-stage renal disease in the United States: an update from the United States Renal Data System. J Am Soc Nephrol 2007; 18: 2644-2648.

8 Levin NW. Intradialytic hypertension: I. Semin Dial 1993; 6: 370-371.

9 Fellner SK. Intradialytic hypertension: II. Semin Dial 1993; 6: 371-373.

10 Inrig JK, Oddone EZ, Hasselblad V, Gillespie B, Patel UD, Reddan D, Toto R, Himmelfarb J, Winchester JF, Stivelman J, Lindsay RM, Szczech LA. Association of intradialytic blood pressure changes with hospitalization and mortality rates in prevalent ESRD patients. Kidney Int 2007; 71: 454-461.

11 Inrig JK, Patel UD, Toto RD, Szczech LA. Association of blood pressure increases during hemodialysis with 2-year mortality in incident hemodialysis patients: a secondary analysis of the Dialysis Morbidity and Mortality Wave 2 Study. Am J Kidney Dis 2009; 54: 881-890.

12 Yang CY, Yang WC, Lin YP. Postdialysis blood pressure rise predicts long-term outcomes in chronic hemodialysis patients: a four-year prospective observational cohort study. BMC Nephrol 2012; 13: 12.

13 Sohn DW, Chai IH, Lee DJ, Kim HC, Kim HS, Oh BH, Lee MM, Park YB, Choi YS, Seo JD, Lee YW. Assessment of mitral annulus velocity by Doppler tissue imaging in the evaluation of left ventricular diastolic function. J Am Coll Cardiol 1997; 30: 474-480.

14 Lang RM, Bierig M, Devereux RB, Flachskampf FA, Foster E, Pellikka PA, Picard MH, Roman MJ, Seward J, Shanewise JS, Solomon SD, Spencer KT, Sutton MS, Stewart WJ. Recommendations for chamber quantification: a report from the American Society of Echocardiography's Guidelines and Standards Committee and the Chamber Quantification Writing Group, developed in conjunction with the European Association of Echocardiography, a branch of the European Society of Cardiology. J Am Soc Echocardiogr 2005; 18: $1440-1463$

$15 \mathrm{Kim}$ YJ, Sohn DW. Mitral annulus velocity in the estimation of left ventricular filling pressure: prospective study in 200 patients. J Am Soc Echocardiogr 2000; 13: 980-985.

16 Nagueh SF, Sun H, Kopelen HA, Middleton KJ, Khoury DS. Hemodynamic determinants of the mitral annulus diastolic velocities by tissue Doppler. J Am Coll Cardiol 2001; 37: 278-285.

17 Rabbitts JA, Strom NA, Sawyer JR, Curry TB, Dietz NM, Roberts SK, Kingsley-Berg SM, Charkoudian N. Influence of endogenous angiotensin II on control of sympathetic nerve activity in human dehydration. J Phsyiol 2009; 587: 5441-5449.

18 Bainbridge FA. The influence of venous filling upon the rate of the heart. J Physiol 1915; 50: 65-84.

19 Crystal GJ, Salem MR. The Bainbridge and the "reverse" Bainbridge reflexes: history, physiology, and clinical relevance. Anesth Analg 2012; 114: 520-532.

20 Chou KJ, Lee PT, Chen CL, Chiou CW, Hsu CY, Chung HM, Liu CP, Fang HC. Physiological changes during hemodialysis in patients with intradialysis hypertension. Kidney Int 2006; 69: 1833-1838.

21 Leite-Moreira AF, Correia-Pinto J, Gillebert TC. Afterload induced changes in myocardial relaxation: a mechanism for diastolic dysfunction. Cardiovasc Res 1999; 43: 344-353.

22 Inrig JK. Intradialytic hypertension: a less-recognized cardiovascular complication of hemodialysis. Am J Kidney 2010; 55: 580-589.

23 Dauterman K, Pak PH, Maughan WL, Nussbacher A, Ariê S, Liu CP, Kass DA. Contribution of external forces to left ventricular diastolic pressure. Implications for the clinical use of the Starling law. Ann Intern Med 1995; 122: 737-742.

24 Atherton JJ, Moore TD, Thomson HL, Frenneaux MP. Restrictive left ventricular filling patterns are predictive of diastolic ventricular interaction in chronic heart failure. J Am Coll Cardiol 1998; 31: 413-418.

25 Moore TD, Frenneaux MP, Sas R, Atherton JJ, Morris-Thurgood JA, Smith ER, Tyberg JV, Belenkie I. Ventricular interaction and external constraint account for decreased stroke work during volume loading in CHF. Am J Physiol Heart Circ Physiol 2001; 281: H2385-H2391.

26 Gũnal Al, Karaca I, Celiker H, Ilkay E, Duman S. Paradoxical rise in blood pressure during ultrafiltration is caused by increased cardiac output. J Nephrol 2002; 15: 42-47.

27 Esposito C, Fasoli G, Plati AR, Bellotti N, Conte MM, Cornacchia F, Foschi A, Mazzullo T, Semeraro L, Dal Canton A. Long-term exposure to high glucose up-regulates VCAMinduced endothelial cell adhesiveness to PBMC. Kidney Int 2001; 59: 1842-1849.

28 Thackeray JT, Radziuk J, Harper ME, Suuronen EJ, Ascah KJ, Beanlands RS, Dasilva JN. Sympathetic nervous dysregulation in the absence of systolic left ventricular dysfunction in a rat model of insulin resistance with hyperglycemia. Cardiovasc Diabetol 2011; 10: 75 .

29 Di Daniele N, De Francesco M, Violo L, Spinelli A, Simonetti G. Renal sympathetic nerve ablation for the treatment of difficult-to-control or refractory hypertension in a haemodialysis patient. Nephrol Dial Transplant 2012; 27: 1689-1690.

30 Noori N, Kalantar-Zadeh K, Kovesdy CP, Murali SB, Bross R, Nissenson AR, Kopple JD. Dietary potassium intake and mortality in long-term hemodialysis patients. Am J Kidney Dis 2010; 56: 338-347.

31 Iseki K, Uehara H, Nishime K, Tokuyama K, Yoshihara K, Kinjo K, Shiohira Y, Fukiyama K. Impact of the initial levels of laboratory variables on survival in chronic dialysis patients. Am J Kidney Dis 1996; 28: 541-548.

32 Hwang JC, Wang CT, Chen CA, Chen HC. Hypokalemia is associated with increased mortality rate in chronic hemodialysis patients. Blood Purif 2011; 32: 254-261.

33 Kovesdy CP, Regidor DL, Mehrotra R, Jing J, McAllister CJ, Greenland S, Kopple JD, Kalantar-Zadeh K. Serum and dialysate potassium concentrations and survival in hemodialysis patients. Clin J Am Soc Nephrol 2007; 2: 999-1007.

34 Inrig JK, Van Buren P, Kim C, Vongpatanasin W, Povsic TJ, Toto RD. Probing the mechanisms of intradialytic hypertension: a pilot study targeting endothelial cell dysfunction. Clin J Am Soc Nephrol201: 6 2016-24.

35 Charytan D, Kuntz RE, Mauri L, DeFilippi C. Distribution of coronary artery disease and relation to mortality in asymptomatic hemodialysis patients. Am J Kidney Dis 2007; 49: 409-416.

36 Nagueh SF, Middleton KJ, Kopelen HA, Zoghbi WA, Quiñones MA. Doppler tissue imaging: a noninvasive technique for evaluation of left ventricular relaxation and estimation of filling pressures. J Am Coll Cardiol 1997; 30: 1527-1533.

37 Bhella PS, Pacini EL, Prasad A, Hastings JL, Adams-Huet B, Thomas JD, Grayburn PA Levine $\mathrm{BD}$. Echocardiographic indices do not reliably track changes in left-sided filling pressure in healthy subjects or patients with heart failure with preserved ejection fraction. Circ Cardiovasc Imaging 2011; 4: 482-489. 